\title{
Changes in the Determinism of the Gait Dynamics with the Intervention of a Robotic Walker
}

\author{
Xianglong Wan * and Yoji Yamada \\ Department of Mechanical Systems Engineering, Nagoya University, Furo-cho, Chikusa-ku, Nagoya, \\ Aichi 464-8603, Japan; yoji.yamada@mae.nagoya-u.ac.jp \\ * Correspondence: wan.xianglong@a.mbox.nagoya-u.ac.jp; Tel.: +81-52-789-4502
}

Received: 2 June 2020; Accepted: 15 July 2020; Published: 18 July 2020

\begin{abstract}
Robotic walkers have gradually been developed over the last decade, and their use has caused changes in gait. However, detailed gait analyses during robotic walker-assisted walking have not been performed. In this study, we aim to identify the changes in determinism of gait dynamics owing to the intervention of a robotic walker. (2) Eleven healthy subjects participated in walking experiments under normal walking, rollator-assisted walking, and robotic walker-assisted walking conditions. We analyzed the measured trunk acceleration to derive the gait parameters, local scaling exponent (LSE, from correlation sum), and percentage of determinism (\%DET, from recurrence plot). (3) The walking speed during rollator-assisted walking was significantly lower than that during robotic walker-assisted walking. Changes in the shape of the LSE along the anterior-posterior direction revealed the influence of the robotic walker at an individual level. The changes in \%DET along the anterior-posterior direction were also significantly different between normal walking and robotic walker-assisted walking. (4) The rollator decreased the walking speed in comparison to normal walking. The changed LSE and reduced \%DET imply reduced deterministic patterns and disturbance to the gait dynamics. The robotic walker only affects the gait dynamics in the anterior-posterior direction. Furthermore, the burden on the subjects was reduced during robotic walker-assisted walking.
\end{abstract}

Keywords: nonlinear time series analysis; robotic walker-assisted walking; gait analysis; changes in the determinism of gait dynamics

\section{Introduction}

Mobility aids reduce the fall risk, increase the autonomy, and improve the quality of life of persons with walking difficulties. Wheeled walkers, known as rollators, are widely used to assist in mobility. In the past decade, robotic walkers have gradually been developed and have attracted the interest of many researchers [1-3]. Different from the traditional rollator, the sensors embedded in robotic walkers collect information on the gait, and the in-wheel motors provide assistance during walking. When the user pushes the handle of the robotic walker to operate it, the user's intention becomes significant $[4,5]$. For instance, the weighted-frequency Fourier linear combiner-Fourier linear combiner algorithm was proposed to extract the voluntary user's command in the walker-assisted gait [6]. A robotic walker was proposed for the adjustment of the motion direction and velocity of the user based on signals from infrared sensors [7].

In addition to the user's intention during assisted walking, the gait alternation with or without conventional rollators has also been investigated. In [8], the authors investigated the biomechanical effects of a rollator on the walking patterns of healthy subjects (age: 35 74 years). Their results showed increased hip flexion, reduced ankle dorsiflexion and knee flexion, and reduced ankle and, especially, knee joint moments. The use of a rollator was found to increase the walking speed of elderly subjects 
(mean age: 82 years) [9] and significantly reduce the lower-limb muscle activity of healthy subjects (age: 22 70 years) [10].

A conventional rollator can be considered as a payload to push as it sustains the weight of the user during walking. Human-machine interactions affect the movements of the user and walker during walker-assisted walking. For robotic walkers, the external force is provided by the motors, and its movements can be much more complex than those of a conventional rollator. The gait dynamics of the user might consequently be affected by the movement of the robotic walker. Therefore, the application of robotic walkers could result in issues regarding changes in gait, different from those related to rollator-assisted walking. However, to the best of our knowledge, few studies have been conducted on the gait analysis during robotic walker-assisted walking. An overall reduction in the spatio-temporal parameters, especially the gait speed, was observed during robotic walker-assisted walking [11]. For the development and recommended daily use of robotic walkers, this topic must thoroughly investigated, as also mentioned in [11]. Conventional approaches to reduce the spatio-temporal parameters, such as the analysis of the gait parameters and kinematics, only examine the nature of the gait patterns instead of the inherent dynamical nature of locomotion.

For the walking movement, nonlinear time series analysis offers a way to measure the dynamic properties of gait, such as local dynamic stability (or periodicity), complexity, and determinism. The local dynamic stability is usually expressed by the maximum Lyapunov exponent [12] and maximum Floquet multipliers $[13,14]$, while the gait complexity can be evaluated by multiscale entropy [15] and permutation entropy [16]. The determinism of a time series indicates whether the time series contains a deterministic component or is purely stochastic. A technique was introduced to distinguish between deterministic chaotic and truly random behaviors [17]. The percentage of determinism (\%DET) is a measure use in recurrence quantification analysis (RQA) and is related to the predictability of dynamical systems; a large \%DET value indicates the presence of deterministic patterns in the dynamics of the time series.

Investigations of gait data based on nonlinear time series with or without a robotic walker enable the deduction of useful information regarding the intrinsic dynamical changes. Most of the nonlinear time series analysis-based studies on gait focused on the local dynamic stability using the maximum Lyapunov exponent [18-21]. However, studies on the sensitivity of the maximum Lyapunov exponent indicated that this measure is suitable for assessing the local dynamic stability at a group level [22], but cannot be used to detect small differences at an individual level [23]. Therefore, the difference in the intrinsic dynamics between gait data with and without a robotic walker may not be detected through investigations of the local dynamic stability. Instead, an investigation of the other dynamic properties would be more suitable.

The objective of this study was to explore the changes in the determinism of gait with the intervention of the robotic walker. We hypothesized that the gait dynamics were affected by the movement of the robotic walker. The experiments were conducted under normal walking, rollator-assisted walking, and robotic walker-assisted walking. The correlation sum and \%DET were employed as the tools for analysis. The investigation of the determinism in gait provides insight into the analysis of the gait with rollators and robotic walkers. Moreover, the measurement methods and results of this study will contribute to the understanding of the interactions between users and walkers, and the development of robotic walkers.

\section{Methods}

\subsection{Experimental Devices}

Figure 1a shows a photograph of the robotic walker [24] used in this study. The walker contains two motors (EC 60 flat, Maxon Motor, Sachseln, Switzerland) at the rear wheels to aid the user and built-in encoders to record the rotational velocity of the wheels. A six-axis force sensor (Dynpick WEF-6A500-10-RCD-B, Wacoh Tech Inc., Takaoka, Japan) with a range of $\pm 500 \mathrm{~N}$ in each 
direction was used to measure the interaction forces between the subjects and robotic walker. A simple control method was implemented during assisted walking: The force provided by the two motors in the forward direction was equivalent to the interaction force in the same direction times the assist ratio $\alpha$. Two wireless IMUs (TSND151, ATR-Promotions Co. Ltd., Kyoto, Japan) recorded the triaxial acceleration data during walking. All the data, including the interaction forces, rotational velocities, and acceleration data were transmitted to a PC for further analysis via Bluetooth, as shown in Figure 2.

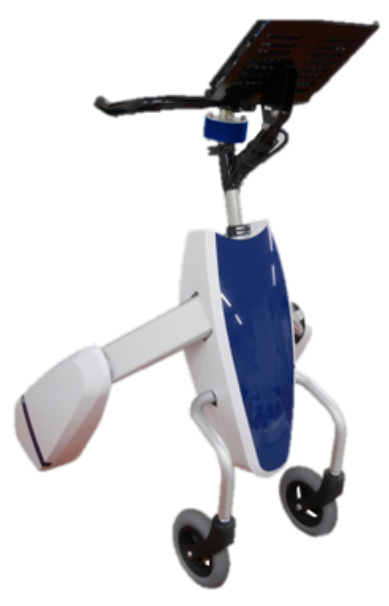

a

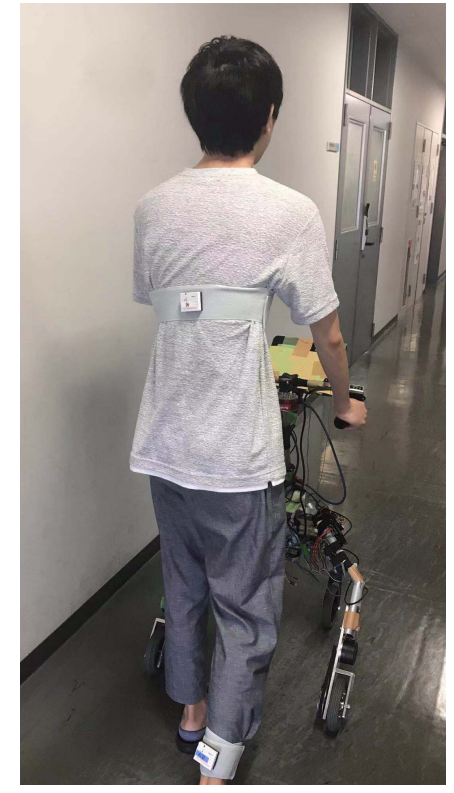

b

Figure 1. Photograph of (a) the robotic walker provided by Panasonic Co., Japan and (b) walking experiment.

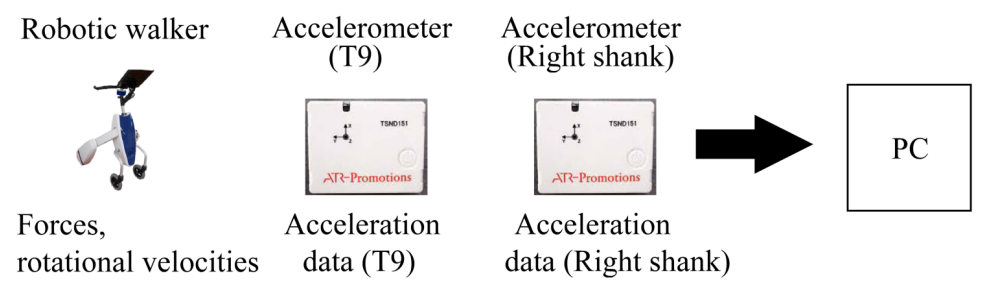

Figure 2. Data measurement process from the robotic walker and accelerometers.

\subsection{Participants and Protocol}

Eleven healthy young males (age: $28.57 \pm 3.3$ years; height: $1.70 \pm 0.01 \mathrm{~m}$; weight: $70.0 \pm 0.02 \mathrm{~kg}$ ) participated in the experiment. None of the participant had any history of injuries in their lower extremities or falls. The subjects signed informed consent forms and the protocols were approved by the institutional review board of Nagoya University. An a priori power assessment of \%DET between normal and robotic walker-assisted walking revealed that at least nine individuals were needed to provide a minimum of $80 \%$ power to detect differences in the $\% \mathrm{DET}$ along the forward direction.

IMUs were attached to the right leg of each subject on the posterior side of the shank and on the spine at the T9 vertebra level. As shown in Figure 1b, each subject walked along an indoor corridor (4 m wide and $80 \mathrm{~m}$ long) for $1 \mathrm{~min}$ at their preferred walking speed as consistently as possible. Three condition were investigated: Normal walking (NW, condition 1), rollator-assisted walking (RW, condition 2), with $\alpha=0$, and robotic walker-assisted walking (RRW, condition 3) with $\alpha=1$. In condition 2 , the robotic walker acted like a rollator, while the motors were activated to provide assistance in condition 3. No subject reported feeling uncomfortable during assisted walking 
(conditions 2 and 3). Experiments in each condition were repeated three times, and the experiments were performed in a random order. After each 1 min-walking trial, the subjects rested for $1 \mathrm{~min}$ to avoid the effects of fatigue. The acceleration data of the right shank and T9 in the three axes were sampled at a frequency of $100 \mathrm{~Hz}$, and the ranges of the sensors were set to \pm 8 and $\pm 2 \mathrm{~g}$. In each trial with the robotic walker, the average walking speed was calculated from the wheel rotational velocities. In each trial of normal walking, the average walking speed was calculated from the total distance and measured with a measuring wheel against the one-minute time frame.

\subsection{Data Analysis}

We used MATLAB (version 9.2, The MathWorks BV, Natrick, USA) to analyze the collected data. Heel contact events were determined according to the occurrence of the maximal vertical right shank acceleration. Trunk accelerations and measured interaction forces in the anterior-posterior (AP), mediolateral (ML), and vertical $(\mathrm{V})$ directions were determined for further analysis. Thirty strides were extracted, and the data of each stride was time-normalized to 100 samples.

The embedding space for the acceleration data from the sensor at T9 was reconstructed based on the nonlinear time series analysis techniques $[25,26]$. Such finite-dimensional space indicates the sampled system's time evolution and can be used to derive the intrinsic properties of the system. We denote the embedding vector as $\mathbf{X}(t)=\{x(t), x(t+\tau), \ldots, x(t+(m-1) \tau)\}$ from a measured time series, $x(t)$. The time delay $\tau$ is estimated based on the averaged mutual information [27], and the embedding dimension $m$ is determined by the false nearest neighbor algorithm [28].

\subsubsection{Recurrence Quantification Analysis}

In [29], the authors introduced the recurrence plot to visualize the recurrences of dynamical systems. A recurrence is a time when a trajectory returns to the neighborhood of a location previously visited. To draw the recurrence plot of the acceleration data in the embedding space, the component $R(i, j)$ in the recurrence matrix $\boldsymbol{R}$ is denoted by a Heaviside step function:

$$
\begin{aligned}
R(i, j) & =\left\{\begin{array}{lc}
1 & \|\mathbf{X}(i)-\mathbf{X}(j)\| \leq \epsilon \\
0 & \text { otherwise }
\end{array}\right. \\
& =H(\epsilon-\|\mathbf{X}(i)-\mathbf{X}(j)\|),
\end{aligned}
$$

where all components with values below the threshold $\epsilon$ are identified as recurrent points. In this study, the norm was chosen as the Euclidean distance as in [30], and the threshold was set to $10 \%$ of the maximum distance as in [31]. The selection of $\epsilon$ will be discussed in Section 4 .

The \%DET, as one of the RQA measures [32], expresses the ratio of the recurrence points that form the diagonal structures with at least length $l_{\min }$ to all recurrence points as follows:

$$
\% \mathrm{DET}=\frac{\sum_{l=l_{\min }}^{N} l P_{l}}{\sum_{l=1}^{N} l P_{l}},
$$

where $l$ is the length of the diagonal lines, and $P_{l}$ is the corresponding histogram. In this study, the parameter $l_{\min }$ was chosen as 2 .

\subsubsection{Correlation Sum}

As an estimator of the correlation integral, the correlation sum indicates the mean probability that two points in the embedding space at two different times are close [17,33]. It reflects self-similarity, and can be calculated as follows:

$$
C(m, \epsilon)=\frac{2}{N(N-1)} \sum_{i=1}^{N} \sum_{j=i+1}^{N} H(\epsilon-\|\mathbf{X}(i)-\mathbf{X}(j)\|),
$$


where $m$ also expresses the embedding dimension, and the function is highly related to the recurrence plot.

The local slope of the correlation sum, which is also called local scaling exponent (LSE) [12,34], is calculated as follows:

$$
D(m, \epsilon)=\frac{\partial \log C(\epsilon)}{\partial \log \epsilon} \approx \frac{\log C\left(\epsilon_{2}\right)-\log C\left(\epsilon_{1}\right)}{\log \epsilon_{2}-\log \epsilon_{1}} .
$$

The LSEs on different scales $\epsilon$ reveal the local scaling structure of the time series [35].

The correlation dimension is a dynamic invariant measure that characterizes the dynamics of the time series. As the scale decreases, the correlation dimension can be estimated when the LSE reaches a plateau, and the value of the LSE for the plateau remains approximately constant in various embeddings. The existence of such plateaus indicates determinism. Regions where the LSE is constant and the same for all embedding dimensions are called scaling ranges. As suggested in [34], the LSEs of the gaussian white noise with different embedding dimensions in Figure 3a indicate that they are separated from each other and increase monotonically to the embedding dimension as the scale $\epsilon$ decreases; such region is called the noise regime. For such data, the scaling regions are hardly discerned, and the corresponding correlation dimension could not be obtained. Figure $3 \mathrm{~b}$ shows the LSEs for an Henon attractor $\left(x_{i+1}=1-1.4 x_{i}^{2}+0.3 x_{i-1}\right)$ with different embedding dimensions and, with and without additive gaussian white noise. Such attractor has a correlation dimension of 1.26, which can be approximated according to the plateaus for noise free data (solid lines) when $\log \epsilon<-4$. For large $\epsilon$ (such as $\log \epsilon>-1$ in the figure), the LSE does not exhibit scaling in this region (macroscopic regime), which only corresponds to the macroscopic structures. When the noise exists (dotted lines) and $\log \epsilon<-1.5$, the LSE reaches a plateau (scaling range) in each embedding. However, the values of the LSE related to the plateau in these embeddings $(m=2,3,4)$ are different from each other and are larger than 1.26. This implies that the true dimension of such data cannot be extracted when there is noise in the data, such as physiological time series, as also mentioned in [34]. The noise regime appears when $\log \epsilon<-3$, where the LSE increases monotonically to the embedding dimension.

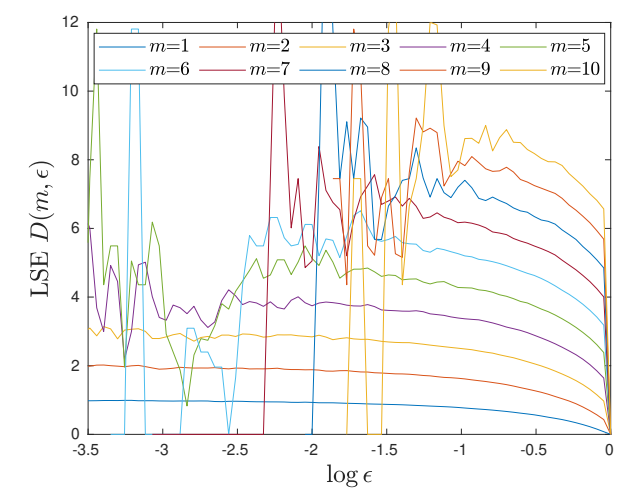

(a)

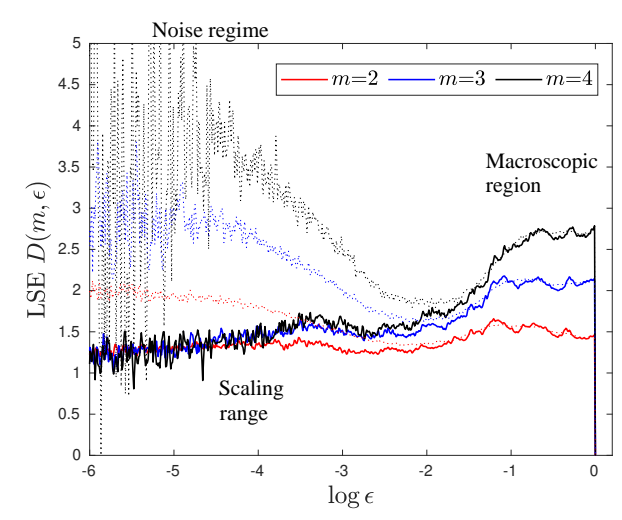

(b)

Figure 3. (a) Local scaling exponents (LSEs) for gaussian white noise with different embedding dimensions, $m=1, \ldots, 10$. (b) LSEs for Henon attractor of length 10,000 with and without $1.5 \%$ additive gaussian white noise; the embedding dimensions are set as $m=2, \ldots, 4$; the solid and dotted lines correspond to the noise free data and data with additive noise, respectively. 


\subsection{Statistical Analysis}

The statistical analysis was performed using MATLAB statistics and machine learning toolbox. ANOVA procedures with post hoc multiple comparison tests were conducted for investigating the statistical differences among these conditions. The Kruskal-Wallis test, which is a non-parametric one-way ANOVA, was first conducted followed by multiple comparison tests if statistically significant differences were detected via the above tests. To determine the difference in the measured interaction forces between conditions 2 and 3, non-parametric Mann-Whitney $U$ tests were also employed. Results with a $p$-value below $0.05(p<0.05)$ were considered statistically significant.

\section{Results}

Table 1 shows the medians of the stride time and walking speed for all the subjects under the three conditions. A Kruskal-Wallis ANOVA showed that there was no statistical difference in stride time among these conditions $(p=0.964)$. However, the Kruskal-Wallis ANOVA rejected the null hypothesis that the walking speeds under all three conditions came from the same distribution $(p=0.018)$. Furthermore, the results of the multiple comparison tests exhibited a significant difference between conditions 1 and $2(p=0.016)$.

Table 1. Measured gait parameters. The values are reported as median \pm median absolute deviation; * indicates a statistically significant difference in walking speed between conditions 1 and 2 .

\begin{tabular}{cccc}
\hline Parameter & Condition 1 NW & Condition 2 RW & Condition 3 RWW \\
\hline Stride time s & $1.15 \pm 0.02$ & $1.15 \pm 0.03$ & $1.16 \pm 0.02$ \\
${\text { Walking speed } \mathrm{m} \cdot \mathrm{s}^{-1}}^{-1.18 \pm 0.04^{*}}$ & $1.13 \pm 0.06^{*}$ & $1.16 \pm 0.04$ \\
\hline
\end{tabular}

The time lag $\tau$ was calculated to be $10 \mathrm{~s}$ for all the subjects, as in [12]. Figure 4a shows the LSEs for the acceleration data of one subject in the AP direction during NW (condition 1). From the figure, it can be seen that the macroscopic regime (approximately $\log \epsilon \geq-0.6$ for $m=5$ ), the LSEs increase as the length scale $\epsilon$ decreases. In the scaling range (approximately $-1.8<\log \epsilon<-0.6$ for $m=5$ ), the LSEs first increase and then decrease. The curves vary slowly around 2 and reach a plateau below 2 . For all embedding dimensions, the values of the LSEs related to the plateau remain approximately constant, while the corresponding scale region shifts with the embedding dimensions. In the noise regime (approximately $\log \epsilon<-1.8$ for $m=5$ ), the LSEs increase as $\epsilon$ increases and reach the embedding dimension at the noise level. This phenomenon was also observed in a previous study [12].

Figure $4 \mathrm{~b}$ shows the LSE curves for the acceleration data of the same subject in the AP direction during RWW (condition 3). It can be seen that, In the macroscopic regime, the curves are similar to the ones in Figure 4a. Significant differences between the two figures can be observed in the scaling range and noise regime. The value of LSE for the plateau becomes larger than 2, and increases slightly with the embedding dimension. In the noise regime, the increment rate of the LSE is larger than that in Figure 4a.

The LSEs for all of the subjects in the AP direction during NW indicated the existence of a plateau in the scaling range; the curves for only two subject were different from those of the others. As shown in Figure 4c, the value of the LSE related to the plateau for one of the two subjects with different LSE increased with the embedding dimension. During assisted walking (conditions 2 and 3), the LSEs for this subject and the plateau vanished (Figure $4 \mathrm{~d}$ ). For the other nine subjects during assisted walking (conditions 2 and 3), individual differences were apparent. The correlation dimensions for seven of them increased with the intervention of the robotic walker, while those for the remaining two subjects were the same as during NW.

Unlike those in the AP direction, the LSEs for the acceleration data of all the subjects in the ML direction are separated from each other, similar to the ones in Figure 4d. The LSEs in the V direction of all the subjects are similar to those in Figure $4 \mathrm{a}$, and the plateaus can be observed under 
all the conditions. This confirms that it is possible to obtain the correlation dimensions. However, the Kruskal-Wallis ANOVA indicates that there is no statistically significant difference in the obtained correlation dimensions along the $\mathrm{V}$ direction under all three conditions $(p=0.61)$. In addition, the values of the LSEs for the plateau in the AP and V directions in condition 2 were slightly higher than those in condition 1.

The embedding dimension $m=5$ was chosen for the calculation of \%DET, while the time lag $\tau$ was set to 10. Table 2 presents the median of \%DETs for all the subjects in the AP, ML, and V directions. In the AP direction, the amount of \%DET is lower when the robotic walker is used. The Kruskal-Wallis ANOVA rejects the null hypothesis that the data in the AP direction under all three conditions comes from the same distribution $(p=0.011)$, while the Kruskal-Wallis ANOVA does not reject the null hypothesis for the \%DETs in the ML and V directions ( $p=0.69$ for the ML direction, and $p=0.84$ for the $\mathrm{V}$ direction). Furthermore, the results of multiple comparison tests shows a significant difference in the median of the \%DETs (AP direction) between conditions 1 and $3(p<0.01)$.

Table 2. \%DETs for the acceleration data of all the subjects in three directions. Values are reported as median \pm median absolute deviation; ${ }^{*}$ indicates a statistically significant difference in the $\% \mathrm{DET}$ between conditions 1 and 3 .

\begin{tabular}{cccc}
\hline Measures & Condition 1 NW & Condition 2 RW & Condition 3 RWW \\
\hline \%DET (AP) & $0.51 \pm 0.02 *$ & $0.49 \pm 0.03$ & $0.46 \pm 0.02 *$ \\
$\%$ DET (ML) & $0.49 \pm 0.08$ & $0.46 \pm 0.05$ & $0.44 \pm 0.07$ \\
$\% D E T(V)$ & $0.68 \pm 0.04$ & $0.69 \pm 0.03$ & $0.68 \pm 0.03$ \\
\hline
\end{tabular}

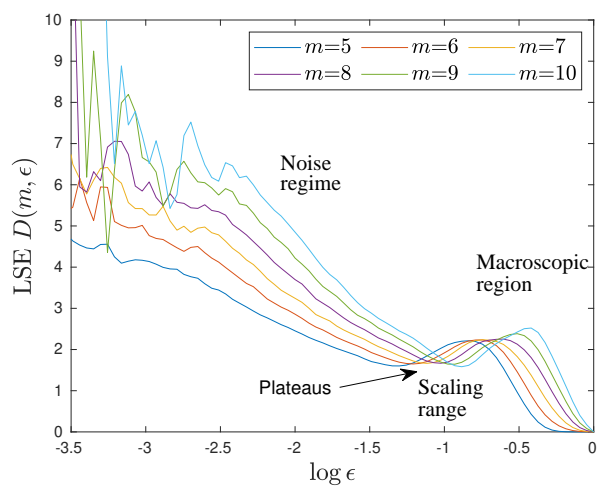

(a)

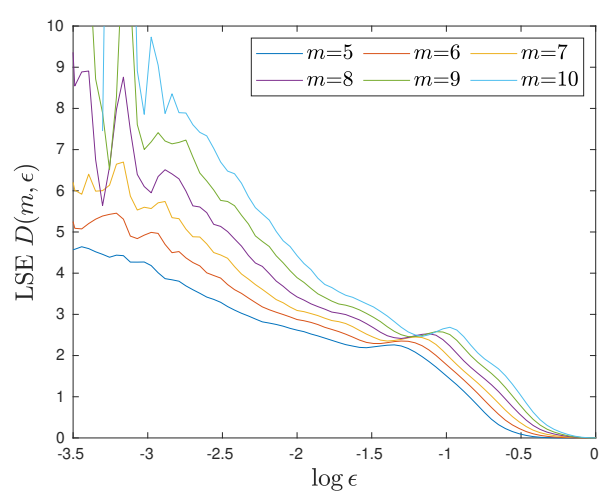

(c)

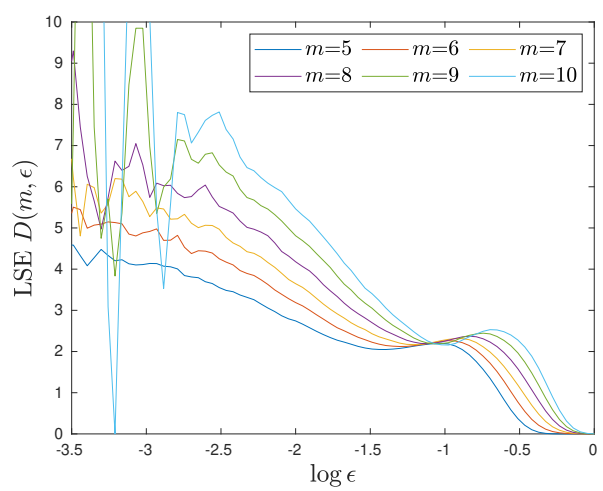

(b)

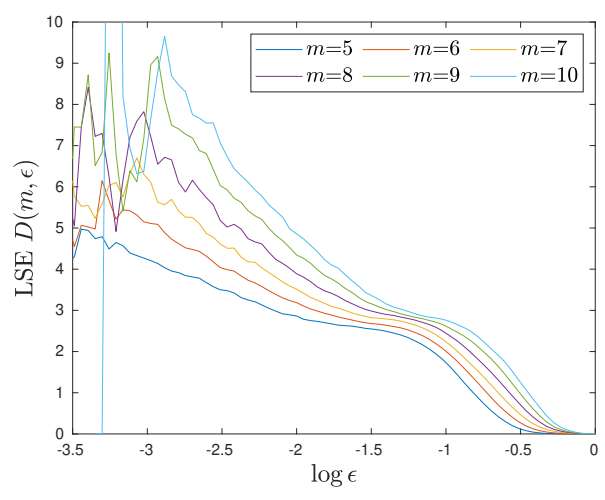

(d)

Figure 4. LSEs of the trunk acceleration data for one subject in the anterior-posterior (AP) direction with different embedding dimensions $(m=5, \ldots, 10)$ in (a) condition 1 and (b) condition 3 . LSEs of the trunk acceleration data for another subject in the AP direction in (c) condition 1 and (d) condition 3. 


\section{Discussion}

\subsection{Gait Alternation with the Intervention of Robotic Walker}

The human gait can be described by a deterministic chaotic system [36,37]. However, diseases such as Parkinson's disease and diabetic peripheral neuropathy result in gait impairments and affect the dynamics of the gait. The results in [38] showed the chaotic nature of the gain of healthy people and the stochastic walking behavior of patients with Parkinson's disease, implying that the chaotic nature was disturbed by the Parkinson's disease. Healthy subjects have been shown to have better local dynamic stability than diabetic neuropathic patients [12].

Similarly, we hypothesized that the gait dynamics would be affected by the movement of the walker. The determinism in gait, as one of the chaotic properties, would change with the intervention of the walkers, including the robotic walker.

We examined the changes in the gait parameters, i.e., the LSE and \%DET, with and without the robotic walker. While no statistical difference was found in the stride time among the three conditions, significant differences were observed in the walking speed between conditions 1 and 2. In condition 2, the walker acts like a payload to push, and its movements slow down the subjects.

The value of the plateau in the scaling range of the LSE can be used to determine the local geometrical structure [33]; if the value remains approximately constant with the increase in the embedding dimension, it could be considered an estimate of the correlation dimension. In general, the existence of the correlation dimension, which is a non-integer, indicates that the intrinsic dynamics are governed by a strange attractor $[17,39]$. As described in Section 3 , even though the plateau in the scaling range exists for the acceleration data (AP direction) of all the subjects during NW, the LSE value related to the plateau for two of them increases with the increase in the embedding dimension (Figure 4c). Thus, the corresponding correlation dimensions for these two subjects in the AP direction could not be estimated using this approach. The totally separated LSEs with different embedding dimensions and vanished plateau (Figure $4 \mathrm{~d}$ ) in the two subjects, indicate that the corresponding behavior related to walker-assisted walking is similar to the noisy one shown in Figure 3a, rather than a pure chaotic one. Thus, the acceleration data during assisted walking seem to be noisier. Moreover, during walker-assisted walking, the shapes of the LSEs of nine subjects in the AP direction remained almost unchanged, while changes in the LSE for the other two subjects were apparent. Consequently, the change in the shape of the LSE of each subject revealed the influence of the walker at an individual level. In the ML direction, because the plateaus were not observed, unlike in the V direction, it is extremely hard to find the deterministic patterns. As shown in Figure 3b, the noise influences the shape of the LSEs. From the change in the shape of the LSE, we noted that the use of the walker results in disturbances to the gait dynamics in the AP direction, which acts like noise. More robust analysis methods are required to obtain the correlation dimensions for all the subjects and conditions cannot be obtained using the current approach.

The $\% \mathrm{DET}$ is the proportion of recurrent points forming diagonal line structures parallel to the main diagonal in the recurrence plot. The \%DET would be very small for purely random processes and equal $100 \%$ for a periodic curve. While the analysis of the LSEs in the AP direction did not exhibit clear, the \%DET in the AP direction were significantly different between NW and RWW. The decrease in \%DET indicated a decrease in the predictability of the gait dynamics in the AP direction. Therefore, the change in \%DET also reflects the influence of the robotic walker in the AP direction. The influence of the rollator was not evident because no significant differences was found between condition 2 and the other conditions. In addition, the much larger median of the \%DET values in the $\mathrm{V}$ direction compared to those of the values in the AP and ML directions indicates that the pseudo-periodic movement in the $\mathrm{V}$ direction is more predictable. In other words, the acceleration data in the $\mathrm{V}$ direction tended to be more regular than those along the other directions in the horizontal plane.

During NW, arms act as passive mass dampers to reduce the torso and head rotation, which is powered by the movement of the lower body [40]. Moreover, the arm swing plays an important role in 
the recovery from a perturbation [41]. During walker-assisted walking (conditions 2 and 3), the hands of the subjects held the handles of the walker to operate it. Thus, the movement of the arms was partially restricted. The interaction forces between the user and walker interfere with the gait dynamics. The forces are first applied to the arm, and then transferred to the upper body indirectly. Therefore, the actual forces applied to the upper body are different from the interaction forces measured and depend on the movements of the arms. Moreover, the user has to recover from disturbances during walker-assisted walking, especially in condition 3.

Figure 5 shows the time histories of the resultant interaction forces in the AP direction in conditions 2 and 3. Negative values indicate that the user pulled the walker during walking. In the case of robotic walker-assisted walking, the driving force comes from both the motor output and the user. It is clear that the average interaction force in condition 2 differs from the one in condition 3 . Then, we calculated the average and standard deviation of the resultant interaction forces in the $\mathrm{AP}, \mathrm{ML}$, and $\mathrm{V}$ directions for all the subjects and carried out Mann-Whitney U tests; the average expresses the overall influence of the force on the subjects and the standard deviation corresponds to the fluctuation. The results of the Mann-Whitney U test indicated that there was no significant difference in these values between conditions 2 and 3, except in the median of the average interaction forces in the AP direction, which is significantly larger in condition 2 than in condition $3(p<0.01)$. The burden on the subjects in the AP direction was reduced during robotic walker-assisted walking. However, the assistance from the robotic walker did not significantly change the fluctuation of the resultant interaction forces. It should be noted that left- and right-side handles are operated by the two arms independently. Forces are transferred from the handles to the trunk and have an overall effect on the fluctuation of the trunk acceleration. In our next work, we will analyze the forces applied to each of the handles, which will give us more information about the interaction between the user and walker.

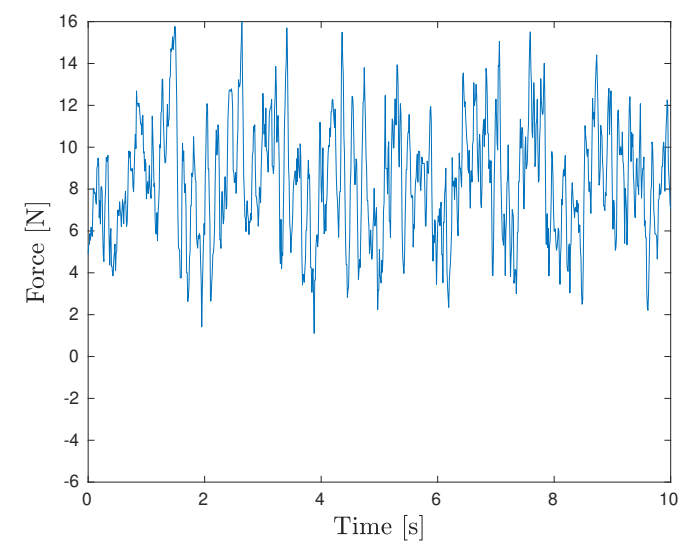

(a)

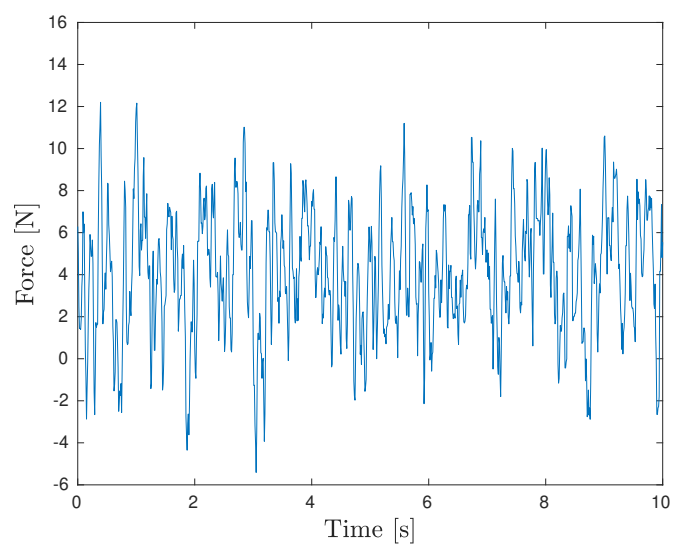

(b)

Figure 5. Time histories of interaction forces in the AP direction during walker-assisted walking in (a) condition 2 and (b) condition 3 .

When the control method described in Section 2.1 was applied, the interaction between the robotic walker and user significantly affected the gait dynamics, and the LSE and \%DET in the AP direction were sensitive to this gait alternation. In comparison to the motion capture system, the overall system is relatively cheap, and applicable in many fields. The calculation of the LSE and \%DET values could be used to asses the control strategy during the development of the robotic walker. If a robotic walker significantly disturbs the gait and results in unstable gait dynamics, the corresponding control method should be improved. The ability of the user to recover from a gait perturbation has been assessed in $[42,43]$. Similarly, the robotic walker and the methods can also be used to assess the capability of resisting perturbations for clinical applications. For instance, participants can walk with a robotic 
walker as a source of perturbation, and the therapists could monitor the changes in the measures with the degree of perturbation.

\subsection{Selection of Threshold}

According to the authors in [44], the selection of an appropriate threshold $\epsilon$ for obtaining the recurrence plot and RQA measures is crucial, and the authors in [45] discussed the importance of keeping the recurrence matrix sparse by selecting a small threshold. The authors in [44] suggested that the threshold should not exceed $10 \%$ of the mean or maximum distance. Another approach for choosing a proper threshold was to seek a scaling region related to the correlation sum [46]. A threshold such that the recurrence point density is approximately $1 \%$ is also available. For the RQA of the gait, a threshold of $40 \%$ and $10 \%$ of the maximum Euclidean distance was chosen in studies $[30,47,48]$ and [49], respectively. In addition, the threshold $\epsilon$ was chosen as the one which corresponds to a fixed sparse recurrence point density in [50].

We calculated the thresholds related to $10 \%$ and $40 \%$ of the maximum distance for all the subjects, with $m=5$ and $\tau=10$. It was found that the values with a $10 \%$ threshold laid in the noise regime, while those with a $40 \%$ threshold were much closer to the macroscopic regime when drawing the LSE curves. When using the 10\% threshold, the recurrence point densities around $1 \%(0.9 \sim 3.5 \%)$ indicated that the corresponding recurrence matrix is sparse. On the other hand, the recurrence point densities (26 73\%) for a $40 \%$ threshold are considered much denser. Similar to the results reported in Section 3, we obtained \%DETs for all the subjects by setting the threshold to $40 \%$ of the maximum distance. No statistical differences among the three conditions were found. Therefore, RQA measures for the $40 \%$ threshold of the maximum distance would not be used to detect the changes in determinism for young healthy subjects with robotic walker, even though evident differences between the adults and toddlers groups were observed in [48]. Instead, a threshold of $10 \%$ of the maximum distance is considered suitable.

\section{Conclusions}

We investigated the influence of a robotic walker on the determinism of the gait dynamics. Eleven healthy young subjects participated in the walking experiment. Three walking conditions were considered, namely normal walking, rollator-assisted walking, and robotic walker-assisted walking. We measured the acceleration of the trunk and used it to calculate the correlation sum and recurrence plot, from which the LSE and \%DET were extracted. The experimental results indicated significant differences in the walking speed between normal walking and rollator-assisted walking. Changes in the shape of the LSE along the AP direction revealed the influence of the robotic walker at an individual level. The \%DET was also significantly different between normal walking and robotic walker-assisted walking in the AP direction. The changes in the LSEs and reduction in \%DET indicated that the movement of the robotic walker affects the gait dynamics only in the AP direction. Compared to the rollator-assisted walking case, the burden on the subjects in the AP direction was reduced during robotic walker-assisted walking. Finally, we also discussed the threshold used for the calculation of recurrence plot and RQA measures.

Author Contributions: Conceptualization, X.W. and Y.Y.; methodology, X.W.; software, X.W.; validation, X.W. and Y.Y.; formal analysis, X.W.; investigation, X.W.; resources, X.W. and Y.Y.; data curation, X.W. and Y.Y.; writing-original draft preparation, X.W.; writing-review and editing, X.W. and Y.Y.; visualization, X.W.; supervision, Y.Y.; project administration, Y.Y.; funding acquisition, Y.Y. Both authors have read and agreed to the published version of the manuscript.

Funding: This research was supported by AMED under Grant Number JP16he1202004.

Conflicts of Interest: The authors declare no conflict of interest.

\section{Abbreviations}


The following abbreviations are used in this manuscript:

$\begin{array}{ll}\text { ANOVA } & \text { Analysis of variance } \\ \text { AP } & \text { Anterior-posterior } \\ \text { IMU } & \text { Inertial measurement unit } \\ \text { LSE } & \text { Local scaling exponent } \\ \text { ML } & \text { Mediolateral } \\ \text { NW } & \text { Normal walking } \\ \text { RQA } & \text { Recurrence quantification analysis } \\ \text { RW } & \text { Rollator-assisted walking } \\ \text { RWW } & \text { Robotic walker-assisted walking } \\ \text { V } & \text { Vertical } \\ \text { \%DET } & \text { Percentage of determinism }\end{array}$

\section{References}

1. Lim, C.D.; Wang, C.M.; Cheng, C.Y.; Chao, Y.; Tseng, S.H.; Fu, L.C. Sensory cues guided rehabilitation robotic walker realized by depth image-based gait analysis. IEEE Trans. Autom. Sci. Eng. 2015, 13, 171-180. [CrossRef]

2. Mun, K.R.; Yeo, B.B.S.; Guo, Z.; Chung, S.C.; Yu, H. Resistance training using a novel robotic walker for over-ground gait rehabilitation: A preliminary study on healthy subjects. Med Biol. Eng. Comput. 2017, 55, 1873-1881. [CrossRef] [PubMed]

3. Ohnuma, T.; Lee, G.; Chong, N.Y. Development of JARoW-II active robotic walker reflecting pelvic movements while walking. Intell. Serv. Robot. 2017, 10, 95-107. [CrossRef]

4. Paulo, J.; Peixoto, P.; Nunes, U.J. ISR-AIWALKER: Robotic walker for intuitive and safe mobility assistance and gait analysis. IEEE Trans. Hum.-Mach. Syst. 2017, 47, 1110-1122. [CrossRef]

5. Chalvatzaki, G.; Papageorgiou, X.S.; Maragos, P.; Tzafestas, C.S. User-Adaptive Human-Robot Formation Control for an Intelligent Robotic Walker using Augmented Human State Estimation and Pathological Gait Characterization. In Proceedings of the 2018 IEEE/RSJ International Conference on Intelligent Robots and Systems (IROS), Madrid, Spain, 1-5 October 2018; pp. 6016-6022.

6. Neto, A.F.; Gallego, J.A.; Rocon, E.; Pons, J.L.; Ceres, R. Extraction of user's navigation commands from upper body force interaction in walker assisted gait. Biomed. Eng. Online 2010, 9, 37. [CrossRef]

7. Lee, G.; Ohnuma, T.; Chong, N.Y. Design and control of JAIST active robotic walker. Intell. Serv. Robot. 2010, 3, 125-135. [CrossRef]

8. Alkjær, T.; Larsen, P.K.; Pedersen, G.; Nielsen, L.H.; Simonsen, E.B. Biomechanical analysis of rollator walking. Biomed. Eng. Online 2006, 5, 2. [CrossRef]

9. Mahoney, J.; Euhardy, R.; Carnes, M. A Comparison of a Two-Wheeled Walker and a Three-Wheeled Walker in a Geriatric Population. J. Am. Geriatr. Soc. 1992, 40, 208-212. [CrossRef]

10. Suica, Z.; Romkes, J.; Tal, A.; Maguire, C. Walking with a four wheeled walker (rollator) significantly reduces EMG lower-limb muscle activity in healthy subjects. J. Bodyw. Mov. Ther. 2016, 20, 65-73. [CrossRef]

11. Frizera, A.; Elias, A.; Del-Ama, A.J.; Ceres, R.; Bastos, T.F. Characterization of spatio-temporal parameters of human gait assisted by a robotic walker. In Proceedings of the 2012 4th IEEE RAS \& EMBS International Conference on Biomedical Robotics and Biomechatronics (BioRob), Rome, Italy, 24-27 June 2012; pp. 1087-1091.

12. Dingwell, J.B.; Cusumano, J.P. Nonlinear time series analysis of normal and pathological human walking. CHAOS 2000, 10, 848-863. [CrossRef]

13. Dingwell, J.B.; Kang, H.G.; Marin, L.C. The effects of sensory loss and walking speed on the orbital dynamic stability of human walking. J. Biomech. 2007, 40, 1723-1730. [CrossRef]

14. Chinimilli, P.T.; Sorkhabadi, S.M.R.; Zhang, W. Assessment of Human Dynamic Gait Stability with a Lower Extremity Assistive Device. IEEE Trans. Neural Syst. Rehabil. Eng. 2020, 28, 669-678. [CrossRef] [PubMed]

15. Costa, M.; Goldberger, A.L.; Peng, C.K. Multiscale entropy analysis of complex physiologic time series. Phys. Rev. Lett. 2002, 89, 068102. [CrossRef] [PubMed]

16. Leverick, G.; Szturm, T.; Wu, C.Q. Using entropy measures to characterize human locomotion. J. Biomech. Eng.-Trans. ASME 2014, 136, 121002. [CrossRef] 
17. Grassberger, P.; Procaccia, I. Characterization of strange attractors. Phys. Rev. Lett. 1983, 50, $346-349$. [CrossRef]

18. Buzzi, U.H.; Stergiou, N.; Kurz, M.J.; Hageman, P.A.; Heidel, J. Nonlinear dynamics indicates aging affects variability during gait. Clin. Biomech. 2003, 18, 435-443. [CrossRef]

19. England, S.A.; Granata, K.P. The influence of gait speed on local dynamic stability of walking. Gait Posture 2003, 25, 172-178. [CrossRef]

20. Caronni, A.; Gervasoni, E.; Ferrarin, M.; Anastasi, D.; Brichetto, G.; Confalonieri, P.; Di Giovanni, R.; Prosperini, L.; Tacchino, A.; Solaro, C.; et al. Local dynamic stability of gait in people with early multiple sclerosis and no-to-mild neurological impairment. In IEEE Transactions on Neural Systems and Rehabilitation Engineering; IEEE: Piscataway, NJ, USA, 2020.

21. Fino, P.C. A preliminary study of longitudinal differences in local dynamic stability between recently concussed and healthy athletes during single and dual-task gait. J. Biomech. 2016, 49, 1983-1988. [CrossRef]

22. Sloot, L.H.; Van Schooten, K.S.; Bruijn, S.M.; Kingma, H.; Pijnappels, M.; van Dieën, J.H. Sensitivity of local dynamic stability of over-ground walking to balance impairment due to galvanic vestibular stimulation. Ann. Biomed. Eng. 2011, 39, 1563-1569. [CrossRef]

23. Van Schooten, K.S.; Rispens, S.M.; Pijnappels, M.; Daffertshofer, A.; van Dieen, J.H. Assessing gait stability: The influence of state space reconstruction on inter-and intra-day reliability of local dynamic stability during over-ground walking. J. Biomech. 2013, 46, 137-141. [CrossRef]

24. Yamada, K.; Yokoya, M.; Yamada, Y. Proposal of walking assistant robot for the elderly which is intended for use in home [in Japanese]. IEICE Tech. Rep. 2016, 116, 25-30.

25. Packard, N.H.; Crutchfield, J.P.; Farmer, J.D.; Shaw, R.S. Geometry from a time series. Phys. Rev. Lett. 1980, 45, 712-716. [CrossRef]

26. Takens, F. Detecting strange attractors in turbulence. In Dynamical Systems and Turbulence, Warwick 1980; Springer: Berlin, Germany, 1981; pp. 366-381.

27. Fraser, A.M.; Swinney, H.L. Independent coordinates for strange attractors from mutual information. Phys. Rev. A 1986, 33, 1134-1140. [CrossRef] [PubMed]

28. Kennel, M.B.; Brown, R.; Abarbanel, H.D. Determining embedding dimension for phase-space reconstruction using a geometrical construction. Phys. Rev. A 1992, 45, 3403-3411. [CrossRef]

29. Eckmann, J.; Kamphorst, S.O.; Ruelle, D. Recurrence plots of dynamical systems. World Sci. Ser. Nonlinear Sci. Ser. A 1995, 16, 441-446.

30. Riva, F.; Toebes, M.; Pijnappels, M.; Stagni, R.; Van Dieen, J. Estimating fall risk with inertial sensors using gait stability measures that do not require step detection. Gait Posture 2013, 38, 170-174. [CrossRef]

31. Riley, M.; Balasubramaniam, R.; Turvey, M. Recurrence quantification analysis of postural fluctuations. Gait Posture 1999, 9, 65-78. [CrossRef]

32. Webber, C.L., Jr.; Zbilut, J.P. Dynamical assessment of physiological systems and states using recurrence plot strategies. J. Appl. Physiol. 1994, 76, 965-973. [CrossRef]

33. Kantz, H.; Schreiber, T. Nonlinear Time Series Anal.; Cambridge University Press: Cambridge, UK, 2004; pp. 77-78.

34. Kantz, H.; Schreiber, T. Dimension estimates and physiological data. CHAOS 1995, 5, 143-154. [CrossRef]

35. Casaleggio, A.; Corana, A.; Ridella, S. Correlation dimension estimation from electrocardiograms. Chaos Solitons Fractals 1995, 5, 713-726. [CrossRef]

36. Perc, M. The dynamics of human gait. Eur. J. Phys. 2005, 26, 525-534. [CrossRef]

37. Stergiou, N.; Decker, L.M. Human movement variability, nonlinear dynamics, and pathology: Is there a connection? Hum. Mov. Sci. 2011, 30, 869-888. [CrossRef] [PubMed]

38. Sarbaz, Y.; Towhidkhah, F.; Jafari, A.; Gharibzadeh, S. Do the chaotic features of gait change in Parkinson's disease? J. Theor. Biol. 2012, 307, 160-167. [CrossRef] [PubMed]

39. Grassberger, P.; Procaccia, I. Measuring the strangeness of strange attractors. Phys. D 1983, 9, $189-208$. [CrossRef]

40. Pontzer, H.; Holloway, J.H.; Raichlen, D.A.; Lieberman, D.E. Control and function of arm swing in human walking and running. J. Exp. Biol. 2009, 212, 523-534. [CrossRef]

41. Bruijn, S.M.; Meijer, O.G.; Beek, P.J.; van Dieën, J.H. The effects of arm swing on human gait stability. J. Exp. Biol. 2010, 213, 3945-3952. [CrossRef]

42. Martelli, D.; Luo, L.; Kang, J.; Kang, U.J.; Fahn, S.; Agrawal, S.K. Adaptation of stability during perturbed walking in Parkinson's disease. Sci. Rep. 2017, 7, 1-11. [CrossRef] 
43. Roeles, S.; Rowe, P.; Bruijn, S.; Childs, C.; Tarfali, G.; Steenbrink, F.; Pijnappels, M. Gait stability in response to platform, belt, and sensory perturbations in young and older adults. Med Biol. Eng. Comput. 2018, 56, 2325-2335. [CrossRef]

44. Marwan, N.; Romano, M.C.; Thiel, M.; Kurths, J. Recurrence plots for the analysis of complex systems. Phys. Rep. 2007, 438, 237-329. [CrossRef]

45. Webber, C.L., Jr.; Zbilut, J.P. Recurrence quantification analysis of nonlinear dynamical systems. Tutorials Contemp. Nonlinear Methods Behav. Sci. 2005, 94, 26-94.

46. Zbilut, J.P.; Zaldivar-Comenges, J.M.; Strozzi, F. Recurrence quantification based Liapunov exponents for monitoring divergence in experimental data. Phys. Rev. A 2002, 297, 173-181. [CrossRef]

47. Riva, F.; Bisi, M.C.; Stagni, R. Gait variability and stability measures: Minimum number of strides and within-session reliability. Comput. Biol. Med. 2014, 50, 9-13. [CrossRef]

48. Bisi, M.C.; Riva, F.; Stagni, R. Measures of gait stability: performance on adults and toddlers at the beginning of independent walking. J. Neuroeng. Rehabil. 2014, 11, 131. [CrossRef] [PubMed]

49. Labini, F.S.; Meli, A.; Ivanenko, Y.P.; Tufarelli, D. Recurrence quantification analysis of gait in normal and hypovestibular subjects. Gait Posture 2012, 35, 48-55. [CrossRef] [PubMed]

50. Afsar, O.; Tirnakli, U.; Marwan, N. Recurrence Quantification Analysis at work: Quasi-periodicity based interpretation of gait force profiles for patients with Parkinson disease. Sci. Rep. 2018, 8, 9102. [CrossRef]

(C) 2020 by the authors. Licensee MDPI, Basel, Switzerland. This article is an open access article distributed under the terms and conditions of the Creative Commons Attribution (CC BY) license (http://creativecommons.org/licenses/by/4.0/). 